\title{
The exact solution of the eigenproblem for the parametric down conversion process in the Kerr medium
}

\author{
Goce Chadzitaskos*, Maciej Horowski**, Anatol Odzijewicz**, Agnieszka \\ Tereszkiewicz** 1
}

\author{
*Faculty of Nuclear sciences and Physical Engineering \\ CTU, Brehova 7, CZ-115 19 Praha 1, Czech Republic \\ **Institute of Theoretical Physics \\ University in Białystok
}

Lipowa 41, 15-424 Białystok, Poland

\begin{abstract}
The eigenproblem for a class of Hamiltonians of the parametric down conversion process in the Kerr medium is solved. Some physical characteristics of the system are calculated.
\end{abstract}

\section{Introduction}

During the recent years the parametric down conversion process has been intensively investigated. It is the simplest nonlinear model in which entangled photons (biphotons) are produced, see $\mathrm{P}-\mathrm{L}$. Another intensively studied phenomenon in quantum optics is the Kerr effect, which is the simplest example of a optical bistability, $\mathrm{P}-\mathrm{V}-\mathrm{L}$. In this paper we consider the Hamiltonian which describes both effects simultaneously. Following [J] we chose the special form of the parametric down conversion term and therefore our Hamiltonian is given by (2.3). Using the techniques developed in $\mathrm{O}-\mathrm{H}-\mathrm{T}$, H-O-T, which are strictly related to the theory of orthogonal polynomials, we solve the eigenproblem for Hamiltonian (2.3). This method works only if the parameters $\omega_{1}, \omega_{2}, g$ and $K_{1}, K_{2}$ included in the Hamiltonian, satisfy extra conditions (3.8), and then the solution is given in terms of the Dual Hahn polynomials. In the last section we show the way how to calculate the expecattion values of physical quantities in any state, in particular coherent state and occupation number state. Physical importance of this model required to take in consideration more details, and it will be done in a separate paper.

\footnotetext{
${ }^{1}$ E-mail:**chadzita@br.fjfi.cvut.cz, *aodzijew@labfiz.uwb.edu.pl, horowski@alpha.uwb.edu.pl, tereszk@alpha.uwb.edu.pl
} 


\section{The Hamiltonian of the system}

Let $\mathbf{a}_{1}, \mathbf{a}_{2}$ and $\mathbf{a}_{1}^{*}, \mathbf{a}_{2}^{*}$ be the usual annihilation and creation operators of photons in the mode 1 and 2 . They satisfy the standard commutation relations

$$
\left[\mathbf{a}_{i}, \mathbf{a}_{j}^{*}\right]=\delta_{i j}, \quad\left[\mathbf{a}_{i}, \mathbf{a}_{j}\right]=0, \quad i, j=1,2,
$$

and act in the Hilbert space $\mathcal{H}$ spanned by the Fock basis

$$
\mathcal{B}_{F}:=\left\{\left|n_{1}, n_{2}\right\rangle=\frac{\left(\mathbf{a}_{1}^{*}\right)^{n_{1}}\left(\mathbf{a}_{2}^{*}\right)^{n_{2}}}{\sqrt{n_{1} ! n_{2} !}}|0,0\rangle: n_{1}, n_{2}=0,1, \ldots\right\}
$$

in the usual way. Process of quantum-optical parametric down conversion is described in any Hamiltonian by the term $\mathbf{a}_{1}^{2} \mathbf{a}_{2}^{*}$, if additionally we need an intensity dependent process we must multiply this term by a function of $\mathbf{a}_{1}^{*} \mathbf{a}_{1}$ and $\mathbf{a}_{2}^{*} \mathbf{a}_{2}$. In this paper, due to [J], we consider the system with the amplitude dependent down conversion process described by $g \sqrt{\mathbf{a}_{2}^{*} \mathbf{a}_{2}} \mathbf{a}_{1}^{2} \mathbf{a}_{2}^{*}$, where $g$ is a real constant. The Kerr effect is described by the terms of the form $\left(\mathbf{a}_{i}^{*} \mathbf{a}_{i}\right)^{2}$. Thus in order to take into consideration the free energy of the modes we will study the following Hamiltonian

$$
\mathbf{H}=\omega_{1} \mathbf{a}_{1}^{*} \mathbf{a}_{1}+\omega_{2} \mathbf{a}_{2}^{*} \mathbf{a}_{2}+K_{1}\left(\mathbf{a}_{1}^{*} \mathbf{a}_{1}\right)^{2}+K_{2}\left(\mathbf{a}_{2}^{*} \mathbf{a}_{2}\right)^{2}+g\left(\sqrt{\mathbf{a}_{2}^{*} \mathbf{a}_{2}} \mathbf{a}_{1}^{2} \mathbf{a}_{2}^{*}+\mathbf{a}_{1}^{* 2} \mathbf{a}_{2} \sqrt{\mathbf{a}_{2}^{*} \mathbf{a}_{2}}\right)
$$

where $\omega_{1}, \omega_{2}, K_{1}, K_{2}$ are real constants. It is easy to check that the operator

$$
\mathbf{R}:=\mathbf{a}_{1}^{*} \mathbf{a}_{1}+2 \mathbf{a}_{2}^{*} \mathbf{a}_{2}
$$

and the projective operator $\mathbf{P}$ defined by

$$
\mathbf{P}\left|n_{1}, n_{2}\right\rangle:=\left\{\begin{array}{cc}
0 & \text { if } n_{1} \text { is even } \\
\left|n_{1}, n_{2}\right\rangle & \text { if } n_{1} \text { is odd }
\end{array}\right.
$$

are constants of motion, and each vector of Fock basis $\mathcal{B}_{F}$ is its eigenvector. In order to decompose the Hilbert space $\mathcal{H}$ into invariant subspaces let us notice that for each nonegative integers $M$ and $p \in\{0,1\}$ the subspace

$$
\mathcal{H}_{p, M}:=\operatorname{span}\{|2 k+p, M-k\rangle, k=0,1, \ldots, M\}
$$

is the eigenspace of the operators $\mathbf{R}$ and $\mathbf{P}$, i.e.

$$
\forall|\psi\rangle \in \mathcal{H}_{p, M} \quad \mathbf{R}|\psi\rangle=(2 M+p)|\psi\rangle \quad \mathbf{P}|\psi\rangle=p|\psi\rangle .
$$

Because of this fact the original Fock space can be decomposed

$$
\mathcal{H}=\bigoplus_{\substack{p \in\{0,1\} \\ M \in \mathbb{N} \cup\{0\}}} \mathcal{H}_{p, M}
$$

where, of course, each $\mathcal{H}_{p, M}$ is $\mathbf{H}$-invariant and of finite dimension

$$
\operatorname{dim} \mathcal{H}_{p, M}=M+1
$$


The eigenproblem for the Hamiltonian (2.3) is reduced to the eigenproblems of the finite-dimensional operators $\mathbf{H}_{p, M}:=\left.\mathbf{H}\right|_{\mathcal{H}_{p, M}}$. The matrix form of $\mathbf{H}_{p, M}$ in the basis $\{|2 k+p, M-k\rangle\}_{k=0}^{M}$ is

$$
\mathbf{H}_{p, M}=2 g \cdot\left(\begin{array}{ccccc}
a_{0} & b_{0} & 0 & \cdots & 0 \\
b_{0} & a_{1} & b_{1} & & \vdots \\
0 & b_{1} & a_{2} & \ddots & 0 \\
\vdots & & \ddots & \ddots & b_{M-1} \\
0 & \cdots & 0 & b_{M-1} & a_{M}
\end{array}\right)+C_{p, M} \cdot\left(\begin{array}{ccccc}
1 & 0 & 0 & \cdots & 0 \\
0 & 1 & 0 & & \vdots \\
0 & 0 & 1 & \ddots & 0 \\
\vdots & & \ddots & \ddots & 0 \\
0 & \cdots & 0 & 0 & 1
\end{array}\right),
$$

where

$$
\begin{aligned}
a_{k} & =\frac{k^{2}}{2 g}\left(4 K_{1}+K_{2}\right)+\frac{k}{2 g}\left(2 \omega_{1}-\omega_{2}+4 p K_{1}-2 M K_{2}\right)+M\left(p+\frac{1}{2}\right), \\
b_{k} & =(M-k) \sqrt{(k+1)\left(k+p+\frac{1}{2}\right)},
\end{aligned}
$$

and the constant is

$$
C_{p, M}=-2 g M\left(p+\frac{1}{2}\right)+\omega_{1} p+\omega_{2} M+K_{1} p+K_{2} M^{2}
$$

In the next section we show that under some special conditions for parameters $\omega_{1}, \omega_{2}$, $g, K_{1}, K_{2}$ the first matrix of (2.10) can be diagonalized by the Dual Hahn polynomials theory.

\section{Dual Hahn polynomials and spectrum of the Hamil- tonian}

We start this section with the brief definition and some important properties of the Dual Hahn polynomials [K-S]. For any fixed natural number $N$ and for $\gamma>-1, \delta>-1$ let us define a finite set of real points

$$
\lambda_{l}=l(l+\gamma+\delta+1), \quad l=0,1, \ldots, N .
$$

The Dual Hahn orthonormal polynomials are, by definition, the finite family of the polynomials $\left\{P_{k}\left(\lambda_{l} ; \gamma, \delta, N\right)\right\}_{k=0}^{N}$ of the discrete variable $\lambda_{l}$ satisfying the following three term recurrence formula

$$
\lambda_{l} P_{k}\left(\lambda_{l} ; \gamma, \delta, N\right)=b_{k} P_{k+1}\left(\lambda_{l} ; \gamma, \delta, N\right)+a_{k} P_{k}\left(\lambda_{l} ; \gamma, \delta, N\right)+b_{k-1} P_{k-1}\left(\lambda_{l} ; \gamma, \delta, N\right),
$$

where $a_{k}, b_{k}$ are defined by

$$
\begin{aligned}
& a_{k}=(k+\gamma+1)(N-k)+k(N+\delta+1-k) \\
& b_{k}=\sqrt{(k+1)(k+\gamma+1)(N-k)(N+\delta-k)} .
\end{aligned}
$$


Formula (3.2) must be satisfied for any $k, l=0,1, \ldots, N$. The orthonormality means

$$
\sum_{l=0}^{N} \varrho_{N}(l ; \gamma, \delta) P_{r}\left(\lambda_{l} ; \gamma, \delta, N\right) P_{s}\left(\lambda_{l} ; \gamma, \delta, N\right)=\delta_{r s}
$$

where

$$
\varrho_{N}(l ; \gamma, \delta)=\frac{(2 l+\gamma+\delta+1)(\gamma+1)_{l}(-N)_{l} N !}{(-1)^{l}(l+\gamma+\delta+1)_{N+1}(\delta+1)_{l} l !}
$$

and the Pochhammer-symbol $(a)_{l}$ is defined by the gamma function

$$
(a)_{l}:=\frac{\Gamma(a+l)}{\Gamma(a)} .
$$

Let us note that the Dual Hahn orthonormal polynomials can be expressed in terms of the hypergeometric functions

$$
P_{k}\left(\lambda_{l} ; \gamma, \delta, N\right)=\sqrt{\frac{\Gamma(\gamma+k+1) \Gamma(\delta+N-k+1)}{k !(N-k) ! \Gamma(\gamma+1) \Gamma(\delta+1)}}{ }_{3} F_{2}\left(\begin{array}{c}
-k,-l, l+\gamma+\delta+1 \\
\gamma+1,-N
\end{array} \mid 1\right) .
$$

Now we use this polynomials to solve the eigenproblem of the Hamiltonian (2.10) for the case when $\omega_{1}, \omega_{2}, g, K_{1}, K_{2}$ obey special conditions

$$
\begin{array}{r}
2 \omega_{1}-\omega_{2}-g=0 \\
2 K_{1}+g=0 \\
K_{2}+2 g=0 .
\end{array}
$$

We observe that the substitution

$$
\gamma=p-\frac{1}{2} \quad \delta=0 \quad N=M
$$

realizes the equivalence between the formulae (2.11) and (3.3). Hence the eigenproblem

$$
\mathbf{H}_{p, M}\left|E_{l, p, M}\right\rangle=E_{l, p, M}\left|E_{l, p, M}\right\rangle \quad l=0,1, \ldots M
$$

due to (3.2) is solved in terms of Dual Hahn polynomials,

$$
\begin{aligned}
E_{l, p, M} & =2 g \lambda_{l}-2 g M\left(p+\frac{1}{2}\right)+\omega_{1} p+\omega_{2} M+K_{1} p^{2}+K_{2} M^{2} \\
\left|E_{l, p, M}\right\rangle & =\sqrt{\varrho_{M}\left(l ; p-\frac{1}{2}, 0\right)} \sum_{k=0}^{M} P_{k}\left(\lambda_{l} ; p-\frac{1}{2}, 0 ; M\right)|2 k+p, M-k\rangle .
\end{aligned}
$$

The multiplier on the right hand side of the last formula is chosen to fulfil the orthonormality condition

$$
\left\langle E_{k, p, M} \mid E_{k^{\prime}, p^{\prime}, M^{\prime}}\right\rangle=\delta_{k k^{\prime}} \delta_{p p^{\prime}} \delta_{M M^{\prime}}
$$

what follows from (3.4). We can also express the vectors $|2 k+p, M-k\rangle$ in the basis of the eigenvectors of $\mathbf{H}_{p, M}$ :

$$
|2 k+p, M-k\rangle:=\sum_{l=0}^{M} \sqrt{\varrho_{M}\left(l ; p-\frac{1}{2}, 0\right)} P_{k}\left(\lambda_{l} ; p-\frac{1}{2}, 0 ; M\right)\left|E_{l, p, M}\right\rangle .
$$




\section{Physical characteristics of the system}

In this section we describe the time evolution of expectation values of physically interesting observable on arbitrary state $|\psi\rangle$. To do this, it is important to calculate the matrix elements of the evolution operator $e^{-i \mathbf{H} t}$. Because $\mathcal{H}_{p, M}$ is $\mathbf{H}$ invariant, the only nonvanishing matrix elements of $e^{-i \mathbf{H} t}$ are of the form

$$
\begin{aligned}
\langle 2 l & \left.+p, M-l\left|e^{-i \mathbf{H} t}\right| 2 k+p, M-k\right\rangle= \\
\quad & =\sum_{j=0}^{M} e^{-i t E_{j, p, M}} \varrho_{M}\left(j ; p-\frac{1}{2}, 0\right) P_{l}\left(\lambda_{j} ; p-\frac{1}{2}, 0, M\right) P_{k}\left(\lambda_{j} ; p-\frac{1}{2}, 0, M\right)
\end{aligned}
$$

what follows from (3.14) and (3.10). The decomposition (2.8) leads us to the following resolution of identity operator 1 in $\mathcal{H}$

$$
\mathbf{1}=\sum_{M=0}^{\infty} \sum_{k=0}^{M} \sum_{p=0}^{1}|2 k+p, M-k\rangle\langle 2 k+p, M-k|
$$

Let $\mathbf{X}$ be any observable and $|\psi\rangle$ any arbitrary chosen initial state. Then using four times the resolution (4.2) and (4.1) we obtain that the time evolution of the expectation value of $\mathbf{X}$ in the state $|\psi\rangle$ is given by

$$
\begin{aligned}
\left\langle\psi\left|e^{i \mathbf{H} t} \mathbf{X} e^{-i \mathbf{H} t}\right| \psi\right\rangle= & \sum_{\substack{M_{1}, M_{4}=0 \\
\infty}}^{1} \sum_{p_{1}, p_{4}=0} \sum_{k_{1}, k_{2}=0}^{M_{1}} \sum_{k_{3}, k_{4}=0}^{M_{4}}\left\langle\psi \mid 2 k_{1}+p_{1}, M_{1}-k_{1}\right\rangle \times \\
& \times\left\langle 2 k_{1}+p_{1}, M_{1}-k_{1}\left|e^{i \mathbf{H} t}\right| 2 k_{2}+p_{1}, M_{1}-k_{2}\right\rangle \times \\
& \times\left\langle 2 k_{2}+p_{1}, M_{1}-k_{2} \mid \mathbf{X} 2 k_{3}+p_{4}, M_{4}-k_{3}\right\rangle \times \\
& \times\left\langle 2 k_{3}+p_{4}, M_{4}-k_{3}\left|e^{-i \mathbf{H} t}\right| k_{4}+p_{4}, M_{4}-k_{4}\right\rangle\left\langle k_{4}+p_{4}, M_{4}-k_{4} \mid \psi\right\rangle .
\end{aligned}
$$

Substituting $|\psi\rangle=|2 l+p, M-l\rangle$ in (4.3) and using (4.1) we obtain as a finite sum

$$
\begin{aligned}
& \left\langle 2 l+p, M-l\left|e^{i \mathbf{H} t} \mathbf{X} e^{-i \mathbf{H} t}\right| 2 l+p, M-l\right\rangle= \\
& =\sum_{j_{1}, j_{2}, k_{2}, k_{3}=0}^{M} e^{-2 i t g\left(\lambda_{j_{2}}-\lambda_{j_{1}}\right)} P_{l}\left(\lambda_{j_{1}} ; p-\frac{1}{2}, 0, M\right) P_{k_{2}}\left(\lambda_{j_{1}} ; p-\frac{1}{2}, 0, M\right) \times \\
& \quad \times \varrho_{M}\left(j_{1} ; p-\frac{1}{2}, 0\right) \varrho_{M}\left(j_{2} ; p-\frac{1}{2}, 0\right) P_{k_{3}}\left(\lambda_{j_{2}} ; p-\frac{1}{2}, 0, M\right) P_{l}\left(\lambda_{j_{2}} ; p-\frac{1}{2}, 0, M\right) \times \\
& \quad \times\left\langle 2 k_{2}+p, M-k_{2}|\mathbf{X}| 2 k_{3}+p, M-k_{3}\right\rangle .
\end{aligned}
$$

Moreover, when we put for example $\mathbf{X}(t)=\mathbf{a}_{1}^{*}(t) \mathbf{a}_{1}(t)$, then

$$
\begin{aligned}
\langle 2 l+p, M & \left.-l\left|e^{i \mathbf{H} t} \mathbf{a}_{1}^{*}(t) \mathbf{a}_{1}(t) e^{-i \mathbf{H} t}\right| 2 l+p, M-l\right\rangle= \\
& =p+2 \sum_{j_{1}, j_{2}, k=0}^{M} k e^{-2 i t g\left(\lambda_{j_{2}}-\lambda_{j_{1}}\right)} P_{l}\left(\lambda_{j_{1}} ; p-\frac{1}{2}, 0, M\right) P_{k}\left(\lambda_{j_{1}} ; p-\frac{1}{2}, 0, M\right) \times \\
& \times \varrho_{M}\left(j_{1} ; p-\frac{1}{2}, 0\right) \varrho_{M}\left(j_{2} ; p-\frac{1}{2}, 0\right) P_{k}\left(\lambda_{j_{2}} ; p-\frac{1}{2}, 0, M\right) P_{l}\left(\lambda_{j_{2}} ; p-\frac{1}{2}, 0, M\right) .
\end{aligned}
$$

Now the calculation of the dispersion for this operator is straightforward, too. When

we consider a coherent state $|\psi\rangle=\left|z_{1}, z_{2}\right\rangle=\sum_{n_{1}, n_{2}=0}^{\infty} \frac{z_{1}^{n_{1}} z_{2}^{n_{2}}}{\sqrt{n_{1} ! n_{2} !}}\left|n_{1}, n_{2}\right\rangle$ then (4.3) takes 
the form

$$
\begin{aligned}
& \left\langle z_{1}, z_{2}\left|e^{i \mathbf{H} t} \mathbf{X} e^{-i \mathbf{H} t}\right| z_{1}, z_{2}\right\rangle= \\
& \sum_{M_{1}, M_{4}=0}^{\infty} \sum_{p_{1}, p_{4}=0}^{1} \sum_{k_{1}, k_{2}=0}^{M_{1}} \sum_{k_{3}, k_{4}=0}^{M_{4}} \frac{z_{1}^{p_{1}+p_{4}} z_{2}^{M_{1}+M_{4}}}{\sqrt{\left(2 k_{1}+p_{1}\right) !\left(2 k_{4}+p_{4}\right) !\left(M_{1}-k_{1}\right) !\left(M_{4}-k_{4}\right) !}}\left(\frac{z_{1}^{2}}{z_{2}}\right)^{k_{1}+k_{4}} \times \\
& \times\left\langle 2 k_{1}+p_{1}, M_{1}-k_{1}\left|e^{i \mathbf{H} t}\right| 2 k_{2}+p_{1}, M_{1}-k_{2}\right\rangle \times \\
& \times\left\langle 2 k_{2}+p_{1}, M_{1}-k_{2} \mid \mathbf{X} 2 k_{3}+p_{4}, M_{4}-k_{3}\right\rangle\left\langle 2 k_{3}+p_{4}, M_{4}-k_{3}\left|e^{-i \mathbf{H} t}\right| 2 k_{4}+p_{4}, M_{4}-k_{4}\right\rangle .
\end{aligned}
$$

The formulas (4.3)-(4.6) initialize a new area of investigations and numeric calculations. It is another thing to study this ideas according to the spirit of the theory presented in [H-O-T]. This short paper do not allow us to expand this subject. Because of the physical importance of this theme we will devote to this subject a separate paper.

\section{Acknowledgements}

We would like to thanks J.Tolar and T. Goliski for them interest in the subject. The authors wish to acknowledge partial support of the Ministry of Education of Czech Republic under the research project MSM210000018.

\section{References}

[P-L] V. Peřinová, A.Lukš "Parametric down-conversion experiments with stationary fields", Forstchr. Phys.51, No.2-3, 211-218 (2003)

[P-V-L] V. Peřinová, V. Vrana A.Lukš, "Quantum statistics of displaced Kerr states", Phys. Rev. A,51, No. 3, 2499-2514 (1995)

[H-O-T] M. Horowski, A.Odzijewicz and A.Tereszkiewicz, "Some integrable system in nonlinear quantum optics", J.Math.Phys. 44, (2003) 2, (480-506), arXiv:math-ph/0207031 v1 23 Jul 2002

[O-H-T] A. Odzijewicz, M. Horowski, A.Tereszkiewicz "Integrable multi-boson systems and orthogonal polynomials" J. Phys. A: Math. Gen. 34, (2001) 4353-4376

[K-S] R. Koekoek, R.F.Swarttouw, "The Askey-scheme of hypergeometric orthogonal polynomials and its q-analoue",http://aw.twi.tudelf.nl

[J] I.Jex, G. Drobny, Phys. Rew. A, 47, 3251 (1993) 